\title{
Study of Stability of Non-linear Model with Hyperbolic Secant Function
}

Anas Salim Youns

College of Basic Education

Department of Mathematics

University of Mosul/Mosul/Iraq

Received

26 / 02 / 2018
Abdul Ghafoor Jasim Salim

College of Computers Sciences and Mathematics

Department of Mathematics

University of Mosul/Mosul/Iraq

Accepted

$04 / 06 / 2018$

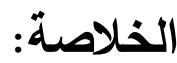

ندرس تعريف جديد لنموذج غير خطي لسلسلة زمنية بدالة القاطع الزائدية في الزمن

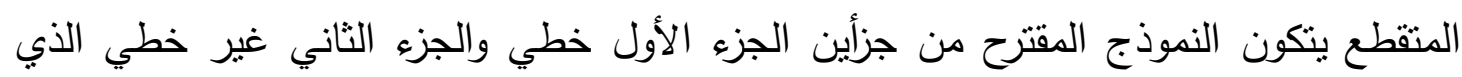

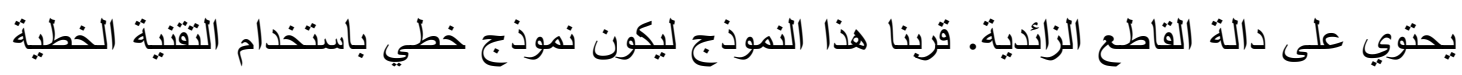

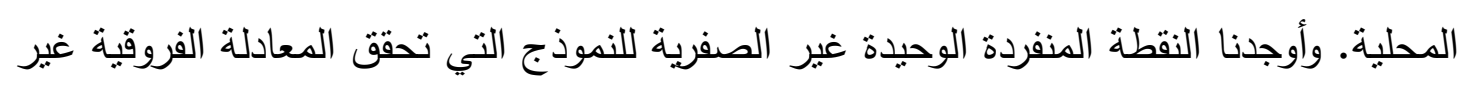

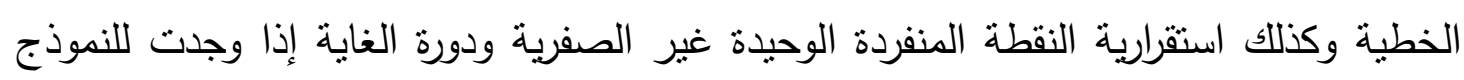

المقترح.

\section{ABSTRACT}

We study a new definition of a non-linear model of time series with hyperbolic secant function in a discrete time. The suggested model consist of two parts the first part is (linear) and the second part is (nonlinear) which contains the hyperbolic secant function. We approximate this model to be linear by using the local linearization technique. We find the non-zero unique singular point that satisfies the non-linear variation equation, also stability of the non-zero unique singular point and the limit cycle if it exists of the suggested model.

Key words. Time series; Local linearization; Singular point; Limit cycle.

\section{Introduction}

We study the non-linear models of time series with hyperbolic secant function. If the roots of the distinctive equation are located in (inside) the unity circle, then the linear (time series) models are stable. Furthermore there are numerous distinct ways to learn the stability for non-linear models. We used the local linearization technique to obtain the approximate linear model near the non-zero unique singular point for 
non-linear model that is suggested in this paper. We find the non-zero unique singular point for the non-linear time series model with hyperbolic secant function, stability for the non-zero unique singular point and the limit cycle if it occurs. Also we find the necessary and enough conditions for the presence, the stability of a non-zero unique singular point and the condition for the stability of the limit cycle if it occurs of the public case of the suggested model.

Finally, we have given some examples (examples(1),(2),) to explain the local linearization technique with a graph of the orbits to show the stable unique singular point and unstable singular point of the suggested model of order one, and example(3) to explain unstable unique singular point of the suggested model of order two with it's graph of the orbits.

\section{Fundamental conceptions for time series}

Definition 2.1: The public linear (autoregressive) model of $p$ order is denoted by $\mathrm{AR}(\mathrm{p})$ that satisfies the next variation equation:

$x_{t}=a_{1} x_{t-1}+\ldots+a_{p} x_{t-p}+z_{t} ; t=0, \mp 1, \mp 2, \ldots$, Where $a_{1}, a_{2}, \ldots, a_{p}$ are the real constants, and $\left\{z_{t}\right\}$ is white noise. The stability state of above linear model that is if the roots of the distinctive equation $\mu^{p}-a_{1} \mu^{p-1}-a_{2} \mu^{p-2} \ldots-a_{p}=0$ are lies in(inside) the unity circle $\left|\mu_{i}\right|<1$ $; \forall i=1,2, \ldots, p[1]$.

Definition 2.2: Let we have the variation equation for the non-linear model that $x_{t}=f\left(x_{t-1}, \ldots, x_{t-p}\right)$, then the non-zero unique singular point $\zeta$ is defined as a point, such that for each of trajectory of the non-linear model $x_{t}=f\left(x_{t-1}, \ldots, x_{t-p}\right)$ beginning to be enough reach it oncoming either if for $t \rightarrow \infty$ or for $t \rightarrow-\infty$. If it (approached) converge it for $t \rightarrow \infty$ then this point $\zeta$ invite as a stable unique singular point and if it (approached)converge for $t \rightarrow-\infty$ then this point $\zeta$ invite as unstable unique singular point. Then to get the unique singular point $\zeta$ we lay that $\zeta=f(\zeta, \ldots, \zeta)$ in the non-linear model $x_{t}=f\left(x_{t-1}, \ldots, x_{t-p}\right)[2]$.

Definition 2.3: The variation equation for a non-linear model $x_{t}=f\left(x_{t-1}, \ldots, x_{t-p}\right)$, then a limit cycle of this model is isolated and closed path $x_{t+1}, \ldots, x_{t+q}$, where $\mathrm{q}$ is a positive integer.

Closed means if the elementary values $\left(x_{1}, \ldots ., x_{p}\right)$ belong to limit cycle then $\left(x_{1+k q}, \ldots, x_{p+k q}\right)=\left(x_{1}, \ldots, x_{p}\right)$ for any integer $\mathrm{k}$. Isolated mean every path beginning enough near the limit cycle converges either for $t \rightarrow \infty$ or for $t \rightarrow-\infty$. When the limit cycle converges it for $t \rightarrow \infty$ we call it a stable limit cycle and if the limit cycle converges it for $t \rightarrow-\infty$ we call it unstable limit cycle [3]. 
Definition 2.4: The exponential model of $\mathrm{p}$ order with appositive parameter $\gamma, \operatorname{expAR}(\mathrm{P})$ is defined by the next variation equation

$$
x_{t}=\left(a_{1}+b_{1} e^{-x_{t-1}^{2}}\right) x_{t-1}+\ldots+\left(a_{p}+b_{p} e^{-x_{t-1}^{2}}\right) x_{t-p}+z_{t}
$$

while $\left\{z_{t}\right\}$ is white noise and $a_{1}, \ldots, a_{p} ; b_{1}, \ldots, b_{p}$ are a parameters of model and $\gamma$ is a positive parameter [4].

The necessary conditions for the presence of the limit cycle of the above model are

1- If all roots for the equation $\mu^{p}-\left(a_{1}\right) \mu^{p-1}-\ldots+\left(a_{p-1}\right) \mu-a_{p}=0$ are located inside the unity circle $\left|\mu_{i}\right|<1$.

2- Some of the roots of the equation $\mu^{p}-\left(a_{1}+b_{1}\right) \mu^{p-1}-\ldots-\left(a_{p-1}+b_{p-1}\right) \mu-\left(a_{p}+b_{p}\right)=0$ lie outside the unity circle $\left|\mu_{i}\right|<1$.

3- The sufficient condition for the limit cycle is $0<\frac{\left(1-\sum_{i=1}^{p} a_{i}\right)}{\left(\sum_{i=1}^{p} b_{i}\right)}<1[5]$.

Definition 2.5: The next non-linear variation (difference) equation $x_{t}=\left(a_{1}+b_{1} e^{-x_{t-1}^{2}}\right) x_{t-1}+\ldots+\left(a_{p}+b_{p} e^{-x_{t-1}^{2}}\right) x_{t-p}+z_{t}$, while $z_{t}$ is white noise and $a_{1}, \ldots, a_{p} ; b_{1}, \ldots, b_{p}$ are the real constants(parameter), then this model is invite as exponential autoregressive model of $p$ order which is denoted by expAR(p) [5].

\section{Theorem 1: [2]}

If $\mathrm{p}=1$ in (definition 2.5), then we get that $x_{t}=\left(a_{1}+b_{1} e^{-x_{t-1}^{2}}\right) x_{t-1}+z_{t}$

The limit cycle of cyclic q $x_{t}, x_{t+1}, x_{t+2}, \ldots, x_{t+q}$ of above model is orbital stable if $\left|\frac{\zeta_{t+q}}{\zeta_{t}}\right|<1$.

\section{Theorem 2: [2]}

For the variation (difference)equation of (definition 2.5) which is not linear that satisfies: $x_{t}=\left(a_{1}+b_{1} e^{-x_{t-1}^{2}}\right) x_{t-1}+\ldots+\left(a_{p}+b_{p} e^{-x_{t-1}^{2}}\right) x_{t-p}+z_{t}$ Then $x_{t}, x_{t+1}, x_{t+2}, \ldots, x_{t+q}$ is called as the limit cycle of cyclic $\mathrm{q}$ of this model which is an orbital stable if all the eigenvalues $\left|\lambda_{i}\right|$ of the matrix $A=A_{q} \cdot A_{q-1} \cdot A_{q-2} \ldots . . A_{2} \cdot A_{1}$ have absolute values lesser than one, such as 


$$
A_{i}=\left(\begin{array}{ccccc}
a_{1}+\left\{b_{1}-2 \sum_{j=1}^{p}\left(b_{j} x_{t+i-j}\right) x_{t+i-1}\right\} e^{-x_{t+i-1}^{2}} & a_{2}+b_{2} e^{-x_{t+i-1}^{2}} \cdot \ldots & . & a_{p}+b_{p} e^{-x_{t+i-1}^{2}} \\
1 & 0 & \ldots & 0 & 0 \\
0 & 1 & \ldots & 0 & 0 \\
\cdot & \cdot & \ldots & . & \cdot \\
\cdot & \cdot & \ldots & . & . \\
\cdot & . & \ldots & . & . \\
\cdot & . & \ldots & . & . \\
0 & 0 & \ldots & 1 & 0
\end{array}\right)
$$

\section{The suggested non-linear model:}

We will give the definition of a non-linear time series model with hyperbolic secant function of order $\mathrm{p}$ such as: $x_{t}=\sum_{i=1}^{p}\left[a_{i}+b_{i} \sec h\left(x_{t-1}\right)\right] x_{t-i}+z_{t}$

Where $\left\{z_{t}\right\}$ is a white noise(normal distribution) and $a_{1}, \ldots, a_{p} ; b_{1}, \ldots, b_{p}$ are the parameters (real constants) of the suggested model.

Note that:

In the non-linear suggested model of an equation (1) we have the next cases:

1) if $x_{t-1} \rightarrow \mp \infty$ then $\sec h\left(x_{t-1}\right) \rightarrow 0$, and the model is linear of order $\mathrm{p}$, that means the model $x_{t}=\sum_{i=1}^{p}\left[a_{i}\right] x_{t-i}+z_{t}$ is linear.

2) Also, if $x_{t-1} \rightarrow 0$, then $\sec h\left(x_{t-1}\right) \rightarrow 1$, and the model is linear of order p, such as: $x_{t}=\sum_{i=1}^{p}\left[a_{i}+b_{i}\right] x_{t-i}+z_{t}$ is linear.

\section{The stability for the suggested model}

In this paragraph, we used the local linearization technique in order to find the stability for non-linear suggested time series models with hyperbolic secant function of order $\mathrm{p}=1,2$, and we generalized this technique to the public model.

The local linearization technique includes three basic steps to find the stability for suggested model namely:

1- The non-zero unique singular point for non-linear model of time series.

2- The stability condition for unique singular point.

3- The stability condition for the limit cycle of the suggest model if it exists.

3.1 Let the non-linear suggested model defined by the next difference equation 


$$
x_{t}=\sum_{i=1}^{p}\left[a_{i}+b_{i} \operatorname{sech}\left(x_{t-1}\right)\right] x_{t-i}+z_{t}
$$

\subsubsection{The unique singular point:}

Let $\mathrm{p}=1$ in variation equation(1), then we have that:

$$
x_{t}=\left[a_{1}+b_{1} \sec h\left(x_{t-1}\right)\right] x_{t-1}+z_{t}
$$

Since, the non-zero unique singular point satisfies that $\zeta=f(\zeta)$ and supposes that the white noise is neglected $\left(z_{t}=0\right)$.

Then, $\zeta=\left[a_{1}+b_{1} \sec h(\zeta)\right] \zeta$, if $(\zeta \neq 0),\left(b_{1} \neq 0\right)$ and $\left(a_{1} \neq 1\right)$.

Then, the non-zero unique singular point

$$
\zeta=\sec h^{-1}\left(\frac{1-a_{1}}{b_{1}}\right), \text { where } 0<\left(\frac{1-a_{1}}{b_{1}}\right) \leq 1
$$

The needful and enough condition for presence of the singular point of the suggested model of one order is that: $0<\left(\frac{1-a_{1}}{b_{1}}\right) \leq 1$.

\subsubsection{The condition of the stable unique singular point:}

We shall find the stability condition for the non-zero unique singular point as next:

Put $x_{s}=\zeta+\zeta_{s}$ for $\mathrm{s}=\mathrm{t}, \mathrm{t}-1$, in the variation equation (2), and let the white noise is zero $z_{t}=0$, then we have:

$\zeta+\zeta_{t}=\left[a_{1}+b_{1} \operatorname{sech}\left(\zeta+\zeta_{t-1}\right)\right]\left(\zeta+\zeta_{t-1}\right)$

Or, $\zeta+\zeta_{t}=\left[a_{1}+b_{1}\left(\frac{2}{e^{\left(\zeta+\zeta_{t-1}\right)}+e^{-\left(\zeta+\zeta_{t-1}\right)}}\right)\right]\left(\zeta+\zeta_{t-1}\right)$

Then, $\zeta_{t}=\left[\left(\frac{a_{1}\left(e^{\zeta}+e^{-\zeta}\right)+2 b_{1}+a_{1} \zeta\left(e^{\zeta}-e^{-\zeta}\right)-\left(e^{\zeta}-e^{-\zeta}\right) \zeta}{\left(e^{\zeta}+e^{-\zeta}\right)}\right)\right] \zeta_{t-1}$

Therefore

$\zeta_{t}=h_{1} \zeta_{t-1}$ where $h_{1}=\left[\left(\frac{a_{1}\left(e^{\zeta}+e^{-\zeta}\right)+2 b_{1}+a_{1} \zeta\left(e^{\zeta}-e^{-\zeta}\right)-\left(e^{\zeta}-e^{-\zeta}\right) \zeta}{\left(e^{\zeta}+e^{-\zeta}\right)}\right)\right]$

The equation (6) is a first order linear model which is stable if the root of the above distinctive equation lies in(inside) the unit circle .

That means if $\left|\mu_{1}\right|=\left|h_{1}\right|<1$.

\subsubsection{The condition for the stable limit cycle if it exists:}

Let the limit cycle of period $\mathrm{q}$ of the suggested model in the variation equation (2) has the form $x_{t}, x_{t+1}, x_{t+2}, \ldots, x_{t+q}=x_{t} \quad$.The points $x_{s}$ near the limit cycle is represented by $x_{s}=x_{s}+\zeta_{s}$, then replace $x_{t}$ and $x_{t-1}$ by $x_{t}+\zeta_{t}$ and $x_{t-1}+\zeta_{t-1}$, and suppose that the white noise is zero $z_{t}=0$. Respectively, then we get:

$x_{t}+\zeta_{t}=\left[a_{1}+b_{1}\left(\frac{2}{e^{\left(x_{t-1}+\zeta_{t-1}\right)}+e^{-\left(x_{t-1}+\zeta_{t-1}\right)}}\right)\right]\left(x_{t-1}+\zeta_{t-1}\right)$ 
Therefore

$$
\zeta_{t}=\left[\left(\frac{a_{1}\left(e^{x_{t-1}}+e^{-x_{t-1}}\right)+2 b_{1}+a_{1} x_{t-1}\left(e^{x_{t-1}}-e^{-x_{t-1}}\right)-\left(e^{x_{t-1}}-e^{-x_{t-1}}\right) x_{t}}{\left(e^{x_{t-1}}+e^{-x_{t-1}}\right)}\right) \zeta_{t-1}\right.
$$

Equation (8) is a first order variation equation with a periodic coefficient, which is not easy to solve analytically. Now, we need to learn that whether $\zeta_{t}$ of equation (8) converges to zero or not, and this can be test by using (Theorem 1), which means $\left|\frac{\zeta_{t+q}}{\xi_{t}}\right|$ is less than one or not [2].

Let, $\mathrm{t}=\mathrm{t}+\mathrm{q}$ in equation (8) we get the following:

$$
\zeta_{t+q}=\left[\left(\frac{a_{1}\left(e^{x_{t+q-1}}+e^{-x_{t+q-1}}\right)+2 b_{1}+a_{1} x_{t+q-1}\left(e^{x_{t+q-1}}-e^{-x_{t+q-1}}\right)-\left(e^{x_{t+q-1}}-e^{-x_{t+q-1}}\right) x_{t+q}}{\left(e^{x_{t+q-1}}+e^{-x_{t+q-1}}\right)}\right) \zeta_{t+q-1}\right.
$$

Hence

$$
\zeta_{t+q}=\prod_{i=1}^{q}\left[\frac{a_{1}\left(e^{x_{t+q-i}}+e^{-x_{t+q-i}}\right)+2 b_{1}+a_{1} x_{t+q-i}\left(e^{x_{t+q-i}}-e^{-x_{t+q-i}}\right)-\left(e^{x_{t+q-i}}-e^{-x_{t+q-i}}\right) x_{t+q-(i-1)}}{\left(e^{x_{t+q-i}}+e^{-x_{t+q-i}}\right)}\right] \zeta_{t}
$$

Then, by (Theorem1) equation (10) is orbitally stable if $\left|\frac{\zeta_{t+q}}{\zeta_{t}}\right|<1$.

Therefore

$\left|\frac{\zeta_{t+q}}{\zeta_{t}}\right|=\left|\prod_{i=1}^{q}\left[\frac{a_{1}\left(e^{x_{t+q-i}}+e^{-x_{t+q-i}}\right)+2 b_{1}+a_{1} x_{t+q-i}\left(e^{x_{t+q-i}}-e^{-x_{t+q-i}}\right)-\left(e^{x_{t+q-i}}-e^{-x_{t+q-i}}\right) x_{t+q-(i-1)}}{\left(e^{x_{t+q-i}}+e^{-x_{t+q-i}}\right)}\right]\right|<1$

Hence, equation (11) can be written equivalently as:

$$
\left|\frac{\zeta_{t+q}}{\zeta_{t}}\right|=\mid \prod_{i=1}^{q}\left[\frac{a_{1}\left(e^{x_{t+i-1}}+e^{-x_{t+i-1}}\right)+2 b_{1}+a_{1} x_{t+i-1}\left(e^{x_{t+i-1}}-e^{-x_{t+i-1}}\right)-\left(e^{x_{t+i-1}}-e^{-x_{t+i-1}}\right) x_{t+i}}{\left(e^{x_{t+i-1}}+e^{-x_{t+i-1}}\right)} \mid<1\right.
$$

\subsection{Substitute $\mathbf{p}=\mathbf{2}$ in equation(1), we get:}

$$
x_{t}=\left[a_{1}+b_{1} \operatorname{sech}\left(x_{t-1}\right)\right] x_{t-1}+\left[a_{2}+b_{2} \operatorname{sech}\left(x_{t-1}\right)\right] x_{t-2}+z_{t}
$$

\subsubsection{The unique singular point:}

By using the definition of the singular point $\zeta=f(\zeta)$ and the white noise be neglected that means $z_{t}=0$.

Then $\zeta=\left[a_{1}+b_{1} \sec h(\zeta)\right] \zeta+\left[a_{2}+b_{2} \sec h(\zeta)\right] \zeta$

The singular point for the variation equation (13):

$$
\zeta=\operatorname{sech}^{-1}\left(\frac{\left(1-\sum_{i=1}^{2} a_{i}\right)}{\left(\sum_{i=1}^{2} b_{i}\right)}\right) \text {, where } 0<\left(\frac{\left(1-\sum_{i=1}^{2} a_{i}\right)}{\left(\sum_{i=1}^{2} b_{i}\right)}\right) \leq 1
$$

The necessary and sufficient condition for the presence of a non-zero unique singular point for the suggested model of two order is that:

$$
0<\left(\frac{1-\sum_{i=1}^{2} a_{i}}{\sum_{i=1}^{2} b_{i}}\right) \leq 1 \text {. }
$$




\subsubsection{The condition of the stable unique singular point:}

We shall find the stability condition for the singular points of the variation equation (13) as follows:

Put $x_{s}=\zeta+\zeta_{s}$ for all $\mathrm{s}=\mathrm{t}, \mathrm{t}-1, \mathrm{t}-2$, and let $z_{t}=0$, then we have:

$\zeta+\zeta_{t}=\left[a_{1}+b_{1}\left(\frac{2}{e^{\left(\zeta+\zeta_{t-1}\right)}+e^{-\left(\zeta+\zeta_{t-1}\right)}}\right)\right]\left(\zeta+\zeta_{t-1}\right)+\left[a_{2}+b_{2}\left(\frac{2}{e^{\left(\zeta+\zeta_{t-1}\right)}+e^{-\left(\zeta+\zeta_{t-1}\right)}}\right)\right]\left(\zeta+\zeta_{t-2}\right)$

Then

$\zeta_{t}=\left[\frac{a_{1}\left(e^{\zeta}+e^{-\zeta}\right)+2 b_{1}+a_{1} \zeta\left(e^{\zeta}-e^{-\zeta}\right)-\left(e^{\zeta}-e^{-\zeta}\right) \zeta+a_{2} \zeta\left(e^{\zeta}-e^{-\zeta}\right)}{\left(e^{\zeta}+e^{-\zeta}\right)}\right] \zeta_{t-1}+\left[\frac{a_{2}\left(e^{\zeta}+e^{-\zeta}\right)+2 b_{2}}{\left(e^{\zeta}+e^{-\zeta}\right)}\right] \zeta_{t-2}$

Or

$$
\zeta_{t}=h_{1} \zeta_{t-1}+h_{2} \zeta_{t-2}
$$

Where

$$
\begin{aligned}
& \left.h_{1}=\frac{a_{1}\left(e^{\zeta}+e^{-\zeta}\right)+2 b_{1}+a_{1} \zeta\left(e^{\zeta}-e^{-\zeta}\right)-\left(e^{\zeta}-e^{-\zeta}\right) \zeta+a_{2} \zeta\left(e^{\zeta}-e^{-\zeta}\right)}{\left(e^{\zeta}+e^{-\zeta}\right)}\right], \\
& h_{2}=\frac{a_{2}\left(e^{\zeta}+e^{-\zeta}\right)+2 b_{2}}{\left(e^{\zeta}+e^{-\zeta}\right)}
\end{aligned}
$$

Then equation (16) is a linear model of order two which has distinctive equation of the form

$$
v^{2}-h_{1} v-h_{2}=0
$$

Then $h_{1}=\left(\mu_{1}+\mu_{2}\right), h_{2}=-\mu_{1} \mu_{2}$

Where $\mu_{1}, \mu_{2}$ are the roots of the distinctive equation of the model.

The stability condition is that $\left|\mu_{i}\right|<1$; for all $\mathrm{i}=1,2$.

\subsubsection{The condition for the stable limit cycle if it exists:}

Let the limit cycle of period $\mathrm{q}$ of the suggested model in the variation equation (13) have the form $x_{t}, x_{t+1}, x_{t+2}, \ldots, x_{t+q}=x_{t}$. The points $x_{s}$ near the limit cycle are represented by $x_{s}=x_{s}+\zeta_{s} ; \forall s=t, t-1, t-2$, and let $z_{t}=0$, then we have

$$
x_{t}+\zeta_{t}=\left[a_{1}+b_{1}\left(\frac{2}{e^{\left(x_{t-1}+\zeta_{t-1}\right)}+e^{-\left(x_{t-1}+\zeta_{t-1}\right)}}\right)\right]\left(x_{t-1}+\zeta_{t-1}\right)+\left[a_{2}+b_{2}\left(\frac{2}{e^{\left(x_{t-1}+\zeta_{t-1}\right)}+e^{-\left(x_{t-1}+\zeta_{t-1}\right)}}\right)\right]\left(x_{t-2}+\zeta_{t-2}\right)
$$

Then

$$
\begin{aligned}
& \zeta_{t}=\left[\frac{a_{1}\left(e^{x_{t-1}}+e^{-x_{t-1}}\right)+2 b_{1}+a_{1} x_{t-1}\left(e^{x_{t-1}}-e^{-x_{t-1}}\right)-\left(e^{x_{t-1}}-e^{-x_{t-1}}\right) x_{t}+a_{2} x_{t-2}\left(e^{x_{t-1}}-e^{-x_{t-1}}\right)}{\left(e^{x_{t-1}}+e^{-x_{t-1}}\right)}\right] \zeta_{t-1} \\
& +\left[\frac{a_{2}\left(e^{x_{t-1}}+e^{-x_{t-1}}\right)+2 b_{2}}{\left(e^{x_{t-1}}+e^{-x_{t-1}}\right)}\right] \zeta_{t-2}
\end{aligned}
$$

Then equation (18) is a 2 nd -order variation equation with a periodic coefficient, which is not easy(difficult) to find it's analytical solution. 
Then we need to know that whether $\zeta_{t}$ of an equation(18) converges to zero or not, and this can be test by using (Theorem 1), that means $\left|\frac{\xi_{t+q}}{\xi_{t}}\right|$ is less than one or not [2].

Suppose that $\mathrm{t}=\mathrm{t}+\mathrm{q}$ in the variation equation (18).

$\zeta_{t+q}=\left[\frac{a_{1}\left(e^{x_{t+q-1}}+e^{-x_{t+q-1}}\right)+2 b_{1}+a_{1} x_{t+q-1}\left(e^{x_{t+q-1}}-e^{-x_{t+q-1}}\right)-\left(e^{x_{t+q-1}}-e^{-x_{t+q-1}}\right) x_{t+q}+a_{2} x_{t+q-2}\left(e^{x_{t+q-1}}-e^{-x_{t+q-1}}\right)}{\left(e^{x_{t+q-1}}+e^{-x_{t+q-1}}\right)}\right] \zeta_{t+q-1}$

$+\left[\frac{a_{2}\left(e^{x_{t+q-1}}+e^{-x_{t+q-1}}\right)+2 b_{2}}{\left(e^{x_{t+q-1}}+e^{-x_{t+q-1}}\right)}\right] \zeta_{t+q-2}$

$\zeta_{t+q}=\left\{\prod_{i=1}^{q}\left[\frac{a_{1}\left(e^{x_{t+q-i}}+e^{-x_{t+q-i}}\right)+2 b_{1}+a_{1} x_{t+q-i}\left(e^{x_{t+q-i}}-e^{-x_{t+q-i}}\right)-\left(e^{x_{t+q-i}}-e^{-x_{t+q-i}}\right) x_{t+q-(i-1)}+a_{2} x_{t+q-(i+1)}\left(e^{x_{t+q-i}}-e^{-x_{t+q-i}}\right)}{\left(e^{x_{t+q-i}}+e^{-x_{t+q-i}}\right)}\right]\right.$

$\left.+\prod_{i=1}^{q-1}\left[\frac{a_{2}\left(e^{x_{t+q-i}}+e^{-x_{t+q-i}}\right)+2 b_{2}}{\left(e^{x_{t+q-1}}+e^{-x_{t+q-i}}\right)}\right]\right\} \zeta_{t}$

Equation (20) is orbitally stable if this condition hold $\left|\frac{\zeta_{t+q}}{\zeta_{t}}\right|<1$.

$\left|\frac{\zeta_{t+q}}{\zeta_{t}}\right|=\mid \prod_{i=1}^{q}\left[\frac{a_{1}\left(e^{x_{t+i-1}}+e^{-x_{t+1-1}}\right)+2 b_{1}+a_{1} x_{t+i-1}\left(e^{x_{t+i-1}}-e^{-x_{t+i-1}}\right)-\left(e^{x_{t+i-1}}-e^{-x_{t+i-1}}\right) x_{t+i}+a_{2} x_{t+i-2}\left(e^{x_{t+i-1}}-e^{-x_{t+i-1}}\right)}{\left(e^{x_{t+q-i}}+e^{-x_{t+q-i}}\right)}\right]$

$+\prod_{i=2}^{q}\left[\frac{a_{2}\left(e^{x_{t i+1-1}}+e^{-x_{t i-1-1}}\right)+2 b_{2}}{\left(e^{x_{t+1-1}}+e^{-x_{t+i-1}}\right)}\right]<1$

\subsection{The stability condition of the public case of the suggested model:}

For the public case for the suggested model.

Let $x_{t}=\sum_{i=1}^{p}\left[a_{i}+b_{i} \operatorname{sech}\left(x_{t-1}\right)\right] x_{t-i}+z_{t}$.

We can find singular point by using the processes which are similar to the equation (3), then we get that:

$\zeta=\sec h^{-1}\left(\frac{\left(1-\sum_{i=1}^{p} a_{i}\right)}{\left(\sum_{i=1}^{p} b_{i}\right)}\right)$, where the necessary and sufficient condition for the presence for the singular point for the suggested model of (p) order is: $0<\left(\frac{1-\sum_{i=1}^{p} a_{i}}{\sum_{i=1}^{p} b_{i}}\right) \leq 1$

The stability for the singular point is 


$$
\begin{aligned}
& \zeta_{t}=\sum_{i=1}^{p} h_{i} \zeta_{t-i}, \text { where } h_{1}=\frac{a_{1}\left(e^{\zeta}+e^{-\zeta}\right)+2 b_{1}+a_{1} \zeta\left(e^{\zeta}-e^{-\zeta}\right)-\left(e^{\zeta}-e^{-\zeta}\right) \zeta+\sum_{i=2}^{p} a_{i} \zeta\left(e^{\zeta}-e^{-\zeta}\right)}{\left(e^{\zeta}+e^{-\zeta}\right)} \\
& h_{i}=\frac{a_{i}\left(e^{\zeta}+e^{-\zeta}\right)+2 b_{i}}{\left(e^{\zeta}+e^{-\zeta}\right)} ; \forall i=2,3, \ldots, p-1, p
\end{aligned}
$$

The stability condition for the singular point of the suggested model of (p) order is satisfied if all the absolute value of the roots $\left|\mu_{i}\right|$ of the distinctive equation $v^{p}-h_{1} v^{p-1}-h_{2} v^{p-2}-h_{3} v^{p-3}-\ldots-h_{p}=0$ must be lie in(inside) the unity circle.

Theorem 3: The model for the variation equation (1), then the limit cycle of period $q, X_{t+1}, X_{t+2}, \ldots, X_{t+q}$ is an orbitally stable when all the eigenvalues of the matrix $A=A_{q} . A_{q-1} \ldots . . A_{1}$ have absolute value less than one, where

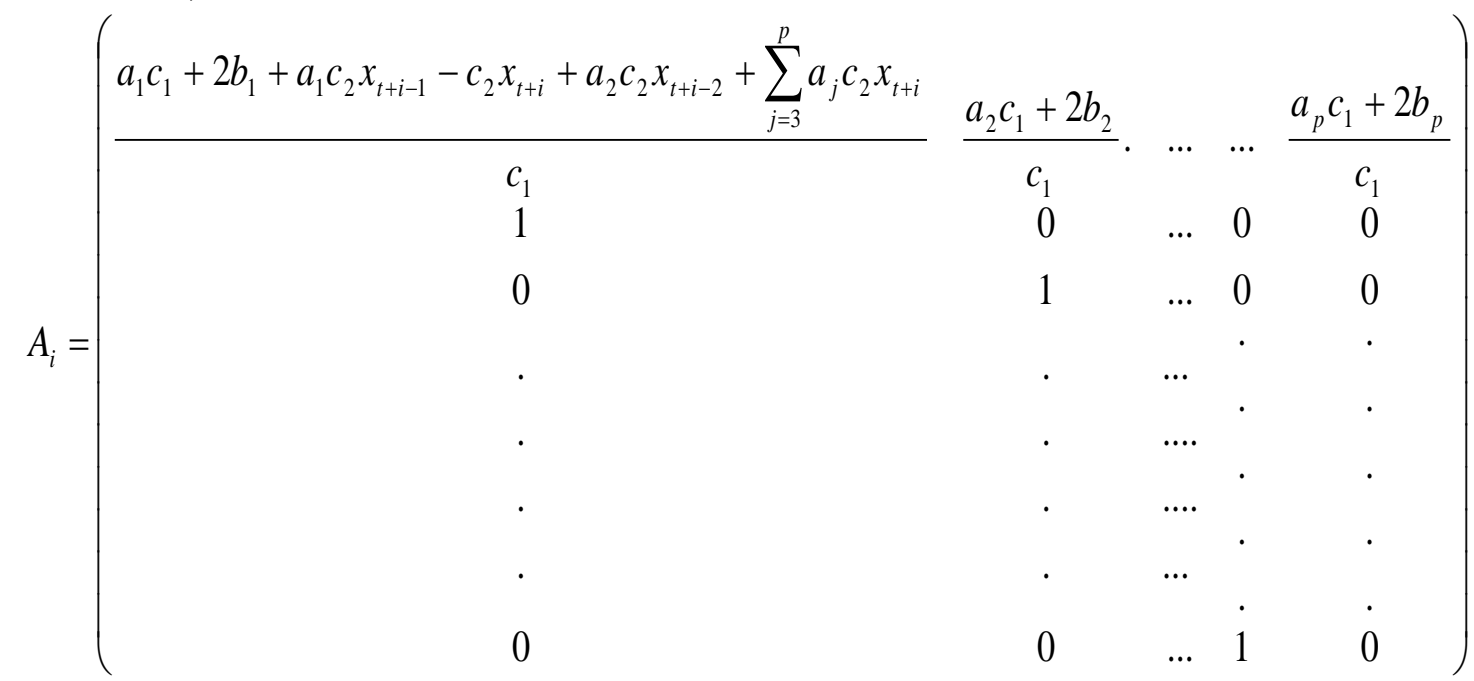

and

$$
\begin{aligned}
& c_{1}=\left(e^{x_{t+i-1}}+e^{-x_{t+i-1}}\right) \\
& c_{2}=\left(e^{x_{t+i-1}}-e^{-x_{t+i-1}}\right)
\end{aligned} .
$$

\section{Examples}

In this paragraph three examples are given to explain how to find the unique singular point for the (non-linear) model of time series, the conditions for stability of unique singular point and graph of the orbits of a non-linear models with hyperbolic secant function of one order and two order.

\section{Example(1):}

Assume that we have the model $x_{t}=\left[-3+5 \sec h\left(x_{t-1}\right)\right] x_{t-1}+z_{t}$, then $a_{1}=-3$ , $b_{1}=5$, Which satisfies the condition that: $0<\left(\frac{1-a_{1}}{b_{1}}\right)=\left(\frac{4}{5}\right) \leq 1$. 
Therefore, $\zeta=\sec h^{-1}\left(\frac{1+3}{5}\right)=\sec h^{-1}(0.8)=0.6931$ is a (non-zero) unique singular point for the model of example(1).

By means of variation equation (6) to get that $\zeta_{t}=-0.6636 \zeta_{t-1}$.

Then, the unique singular point of the example is stable because of the root $\mu=-0.6636$ of the a above variation equation is located inside the unit circle, and the next figures offer the stability of the model of the example(1) for various initial values.

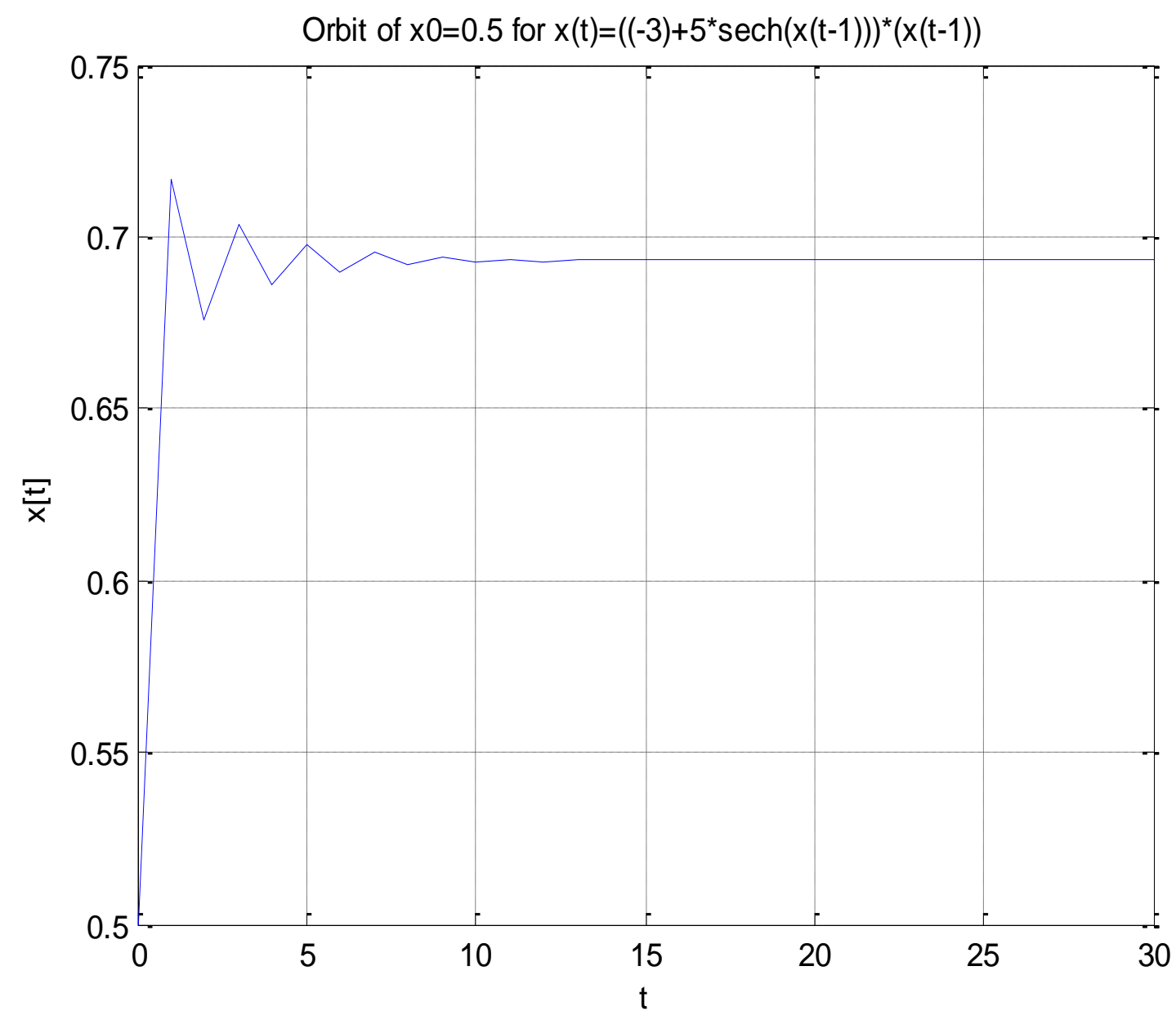

Figure(1) 


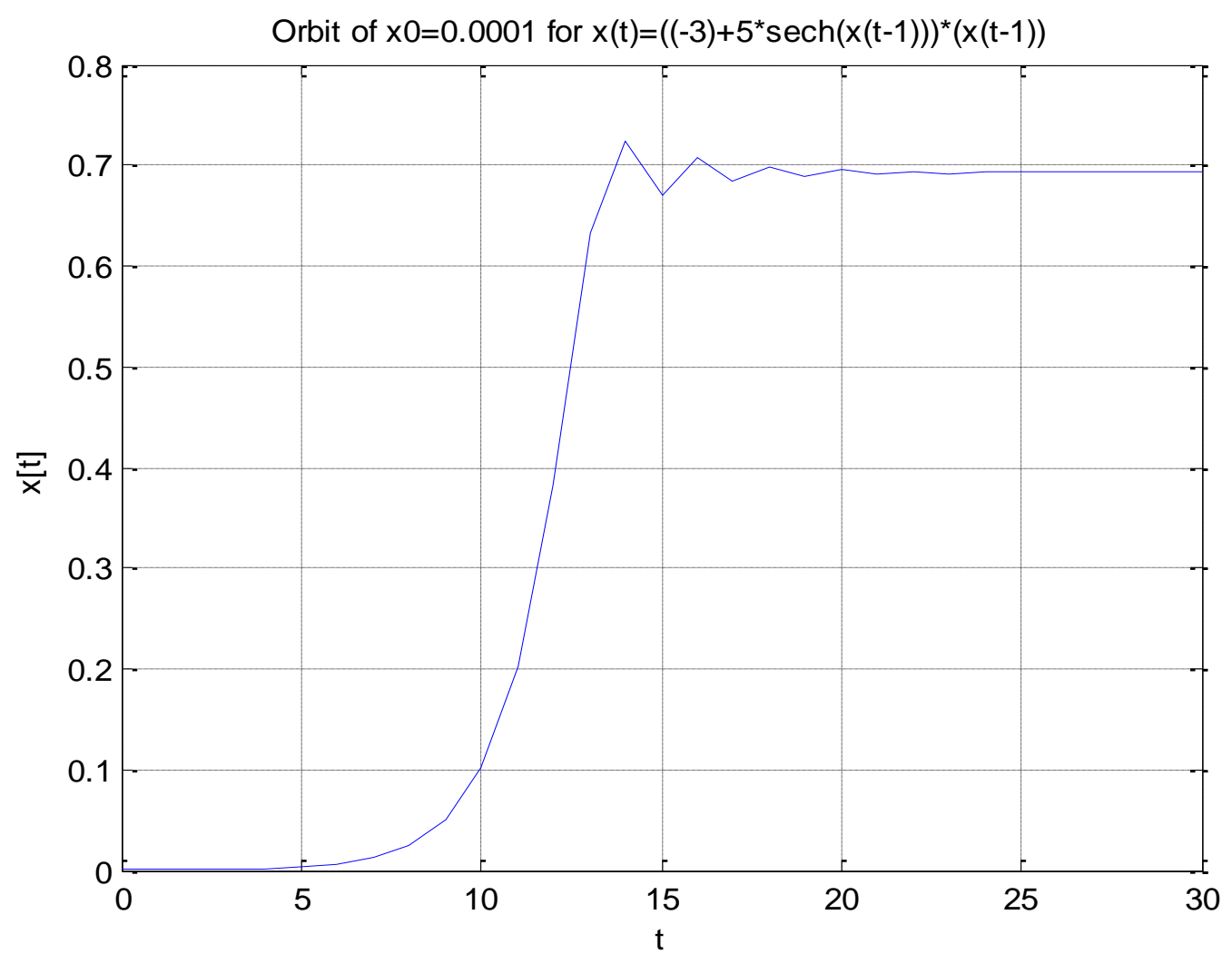

Figure(2)

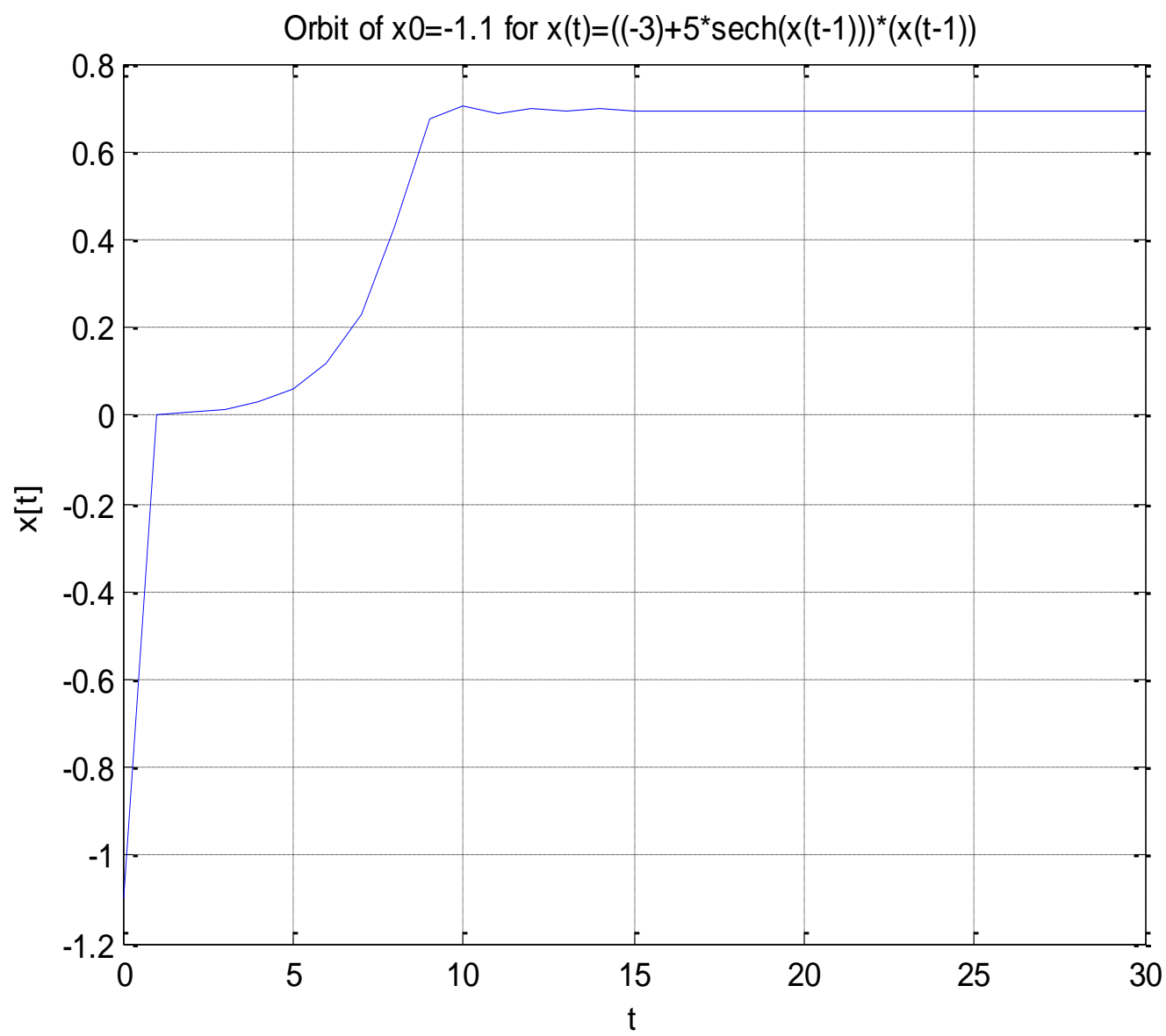

Figure (3) 


\section{Example(2):}

If $a_{1}=-1, b_{1}=20$ then the model in variation equation (2) is $x_{t}=\left[-1+20 \sec h\left(x_{t-1}\right)\right] x_{t-1}+z_{t}$, which satisfies the condition that: $0<\left(\frac{1-a_{1}}{b_{1}}\right)=\left(\frac{2}{20}\right) \leq 1$.

Therefore, $\zeta=\sec h^{-1}\left(\frac{1+1}{20}\right)=\sec h^{-1}(0.1)=2.9932$ is the (non-zero) unique singular point. By applying the variation equation (6), we obtain an equation $\zeta_{t}=-4.9564 \zeta_{t-1}$. Then, the singular point of an example(2) is not stable because the root of the distinctive equation is located outside the unit circle, and the next figures offer that the model of example(2) is unstable for various initial values.

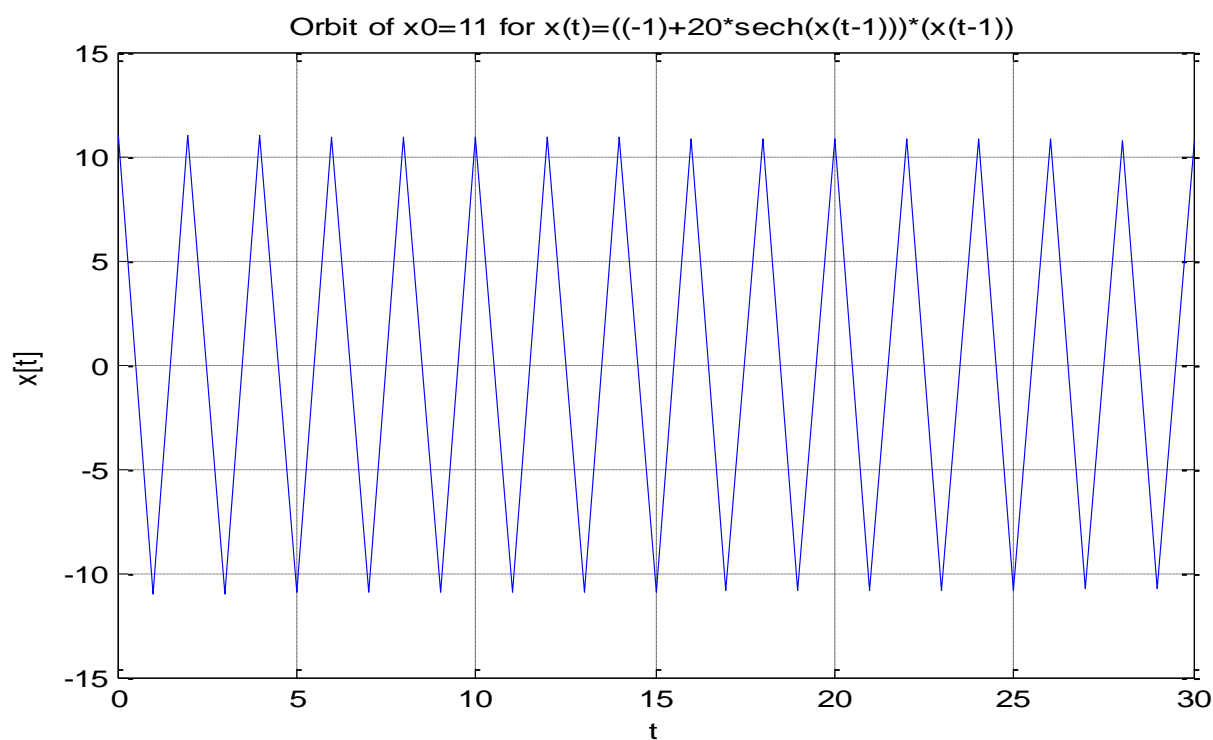

Figure(4)

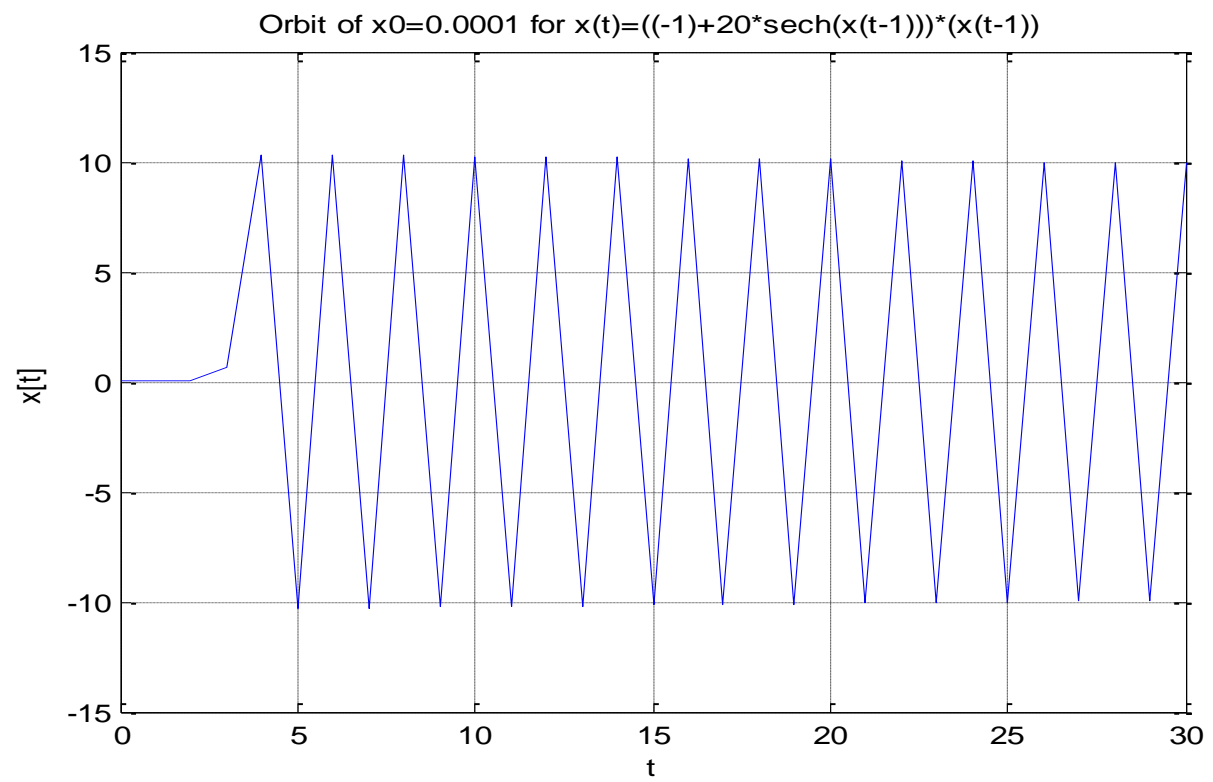

Figure(5) 


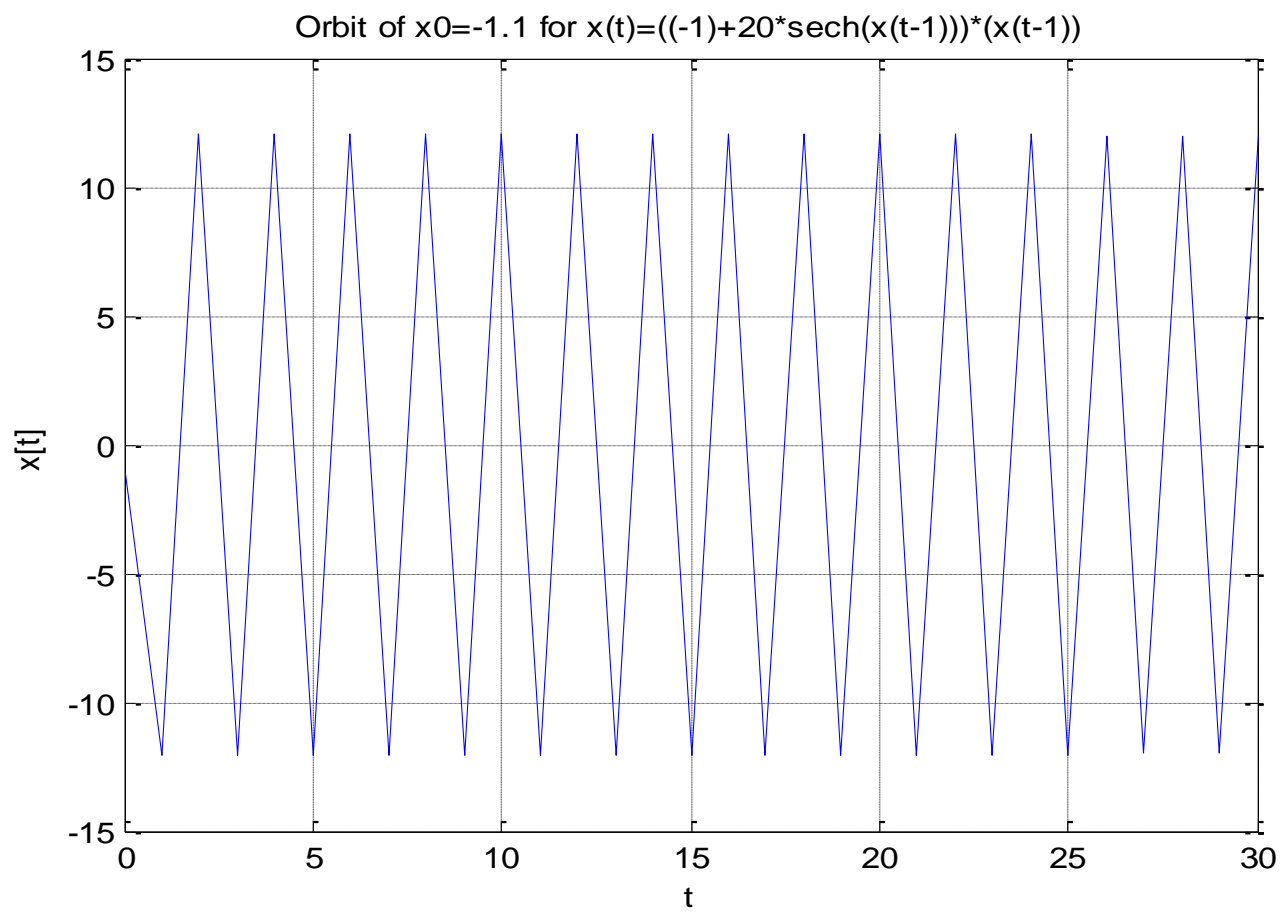

\section{Example(3):}

Figure (6)

If $a_{1}=-2, a_{2}=-3, b_{1}=4, b_{2}=3$ in variation equation(14), then we have the (non-linear) model such that:

$x_{t}=\left[-2+4 \operatorname{sech}\left(x_{t-1}\right)\right] x_{t-1}+\left[-3+3 \operatorname{sech}\left(x_{t-1}\right)\right] x_{t-2}+z_{t}$, which satisfies the condition that:

$0<\left(\frac{1-\sum_{i=1}^{2} a_{i}}{\sum_{i=1}^{2} b_{i}}\right)=\left(\frac{6}{7}\right) \leq 1$

Therefore, $\zeta=\sec h^{-1}\left(\frac{1+5}{7}\right)=\sec h^{-1}\left(\frac{6}{7}\right)=0.5696$ is the (non-zero) unique singular point .

Then, applying variation equation (17), we get that $\zeta_{t}=1.4286 \zeta_{t-1}-0.4286 \zeta_{t-2}$.

The distinctive equation of the linear model is $v^{2}-1.4286 v+0.4286=0$. Then, $\mu_{1}=-1, \mu_{2}=-0.4286$ are the two roots for the distinctive equation of the linear model.

Since the stability conditional of singular point is not satisfied, then the singular point for example (3) is not stable, and the next figures offer that the model of example (3) is not stable(unstable) for various initial values. 


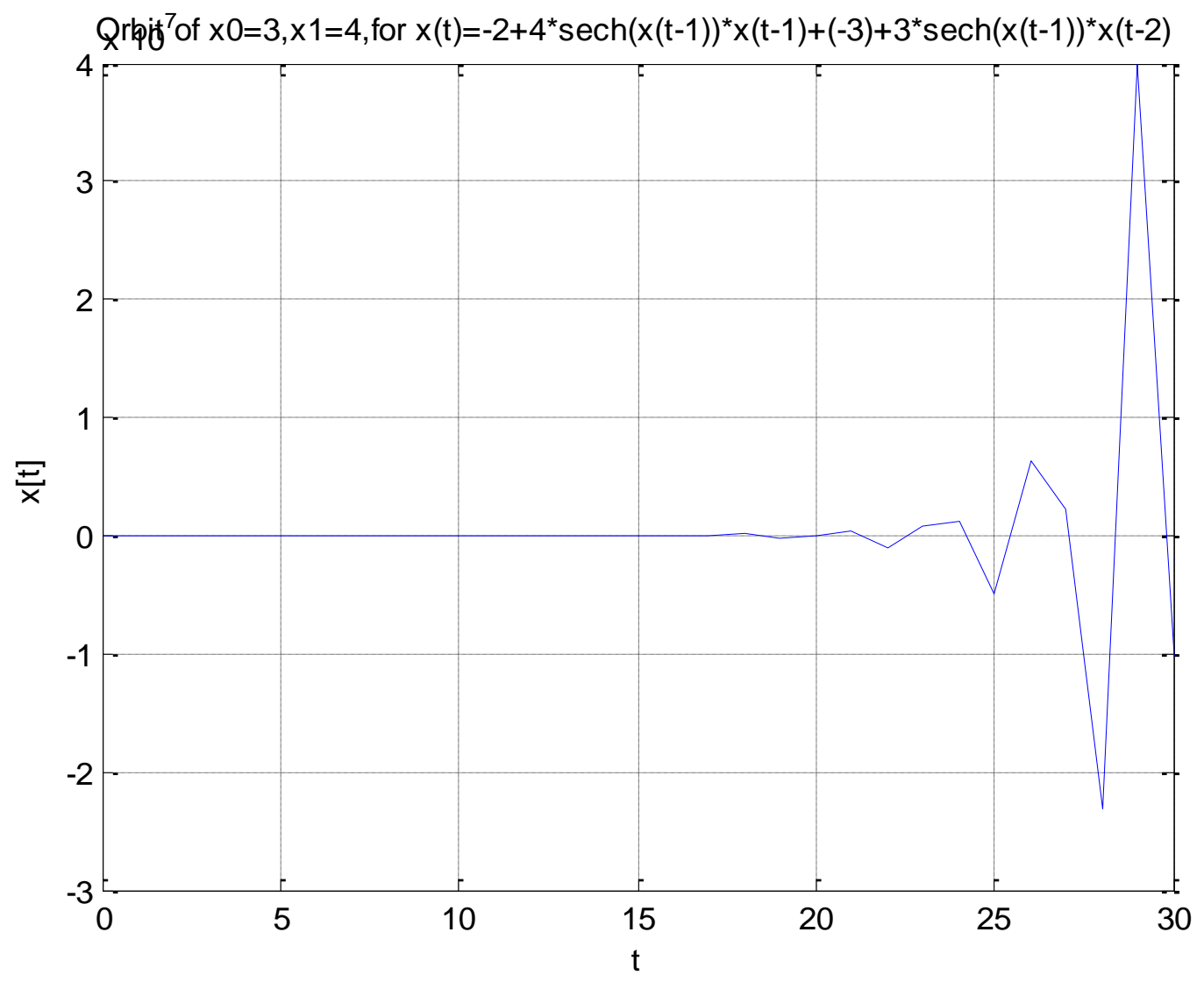

Figure(7)

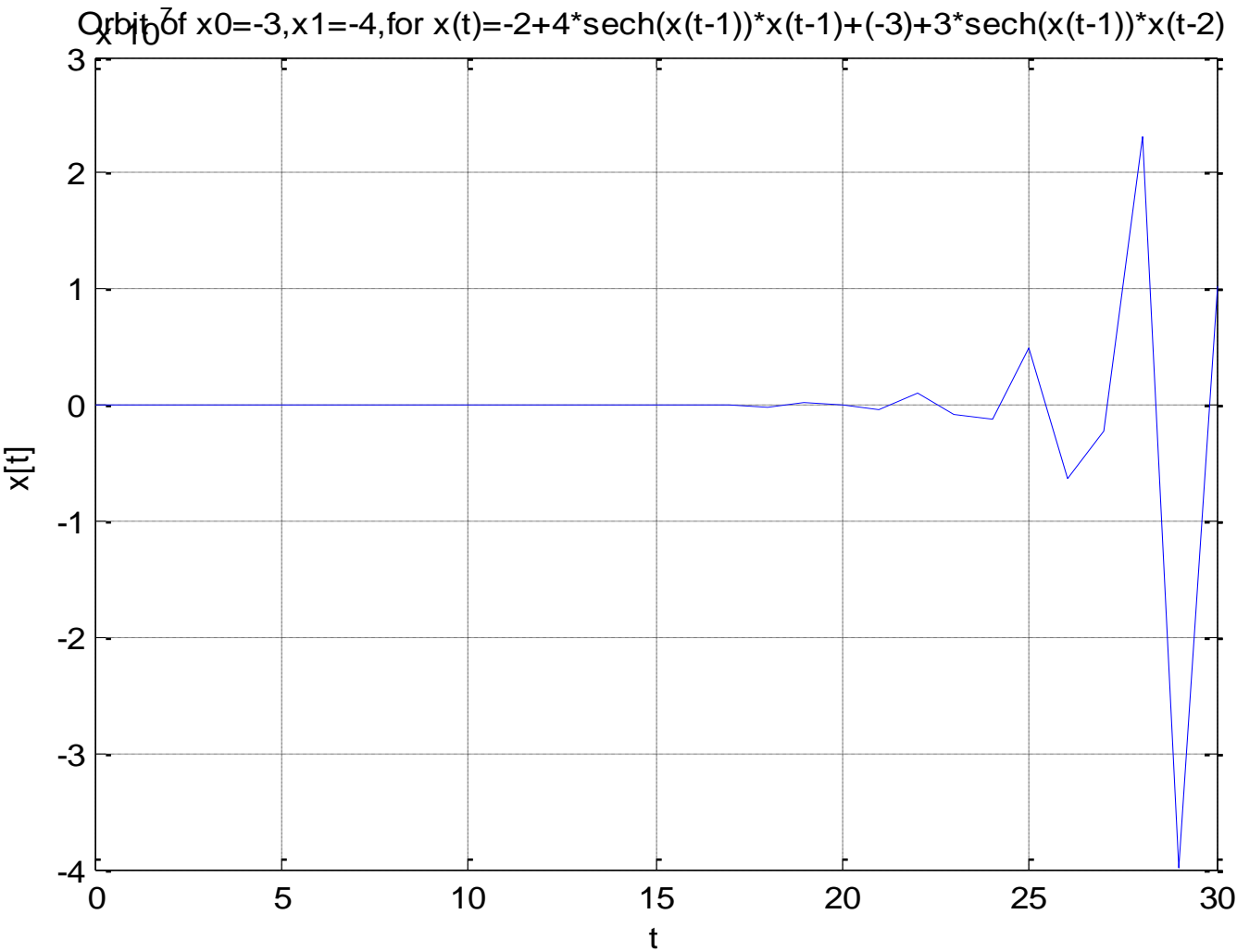

Figure(8) 


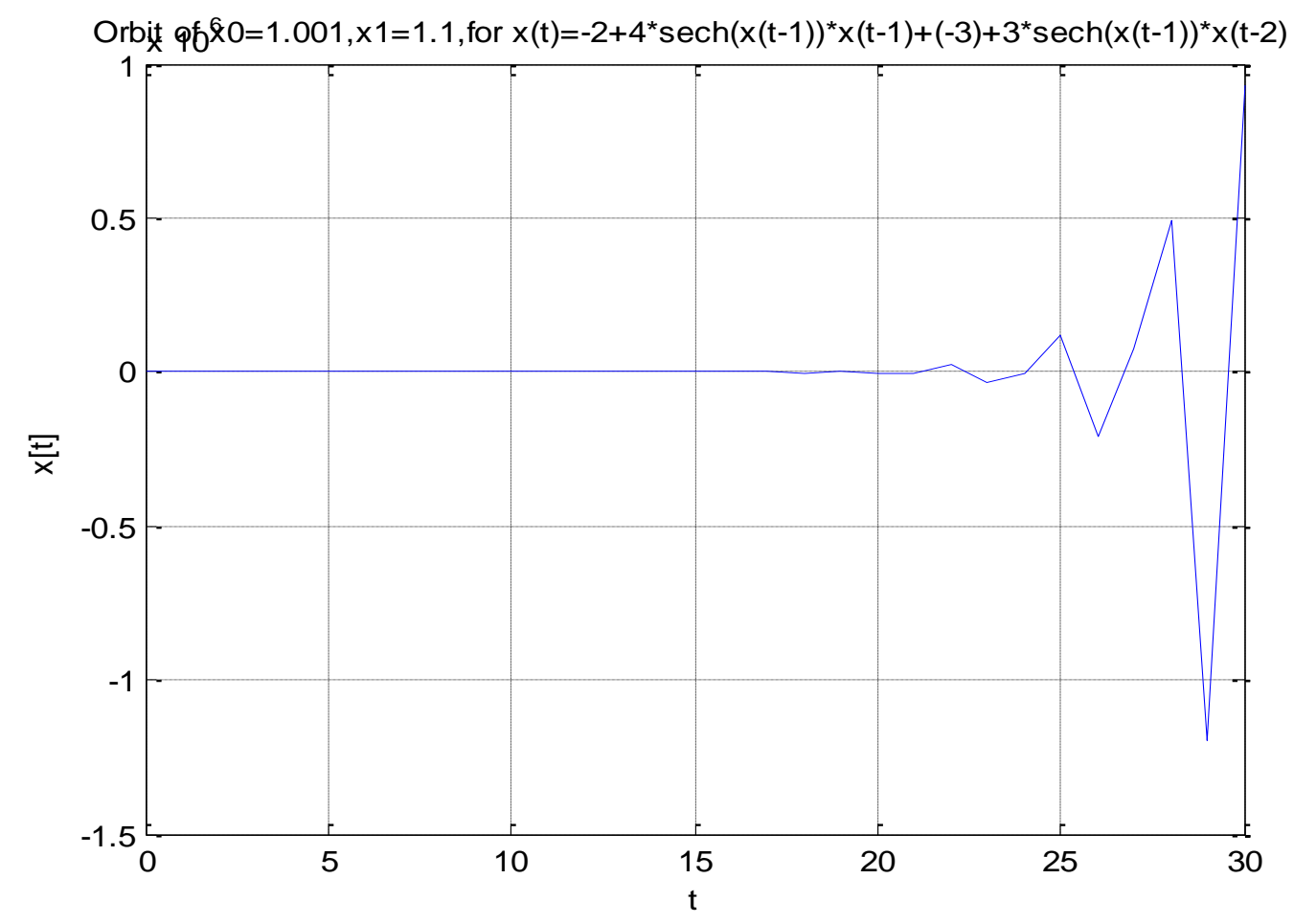

Figure (9)

\section{REFERENCES}

[1] Brockwell, P. J. and Davis, R. A.," Introduction of time series and forecasting". $2^{\text {nd }}$ Ed. Springer-Verlag, New York, Int., U.S.A. . (2002).

[2] Ozaki, T., "Non-linear phenomena and time series model". Invited paper, 43-rd Session of International Statistical Institute, Bulletin of the International Statistical Institute 49, Buenos Aires, Argentina. (1981).

[3] Mohammad A.A. and Salim A.J. ,"Stability of analysis of logistic autoregressive model". Qatar university, Sci. J. ,27,pp 17-28. (2007).

[4] Shi, Z., Tamura, Y., and Ozaki, T. ,"A Study on real-time detecting of machine tool chatter*". International Journal of The Japan Society for Precision Engineering, Vol.32,No.3,pp 178-182. (1998).

[5] Priestley M.B., "Non-linear and non-stationary time series analysis". Academic Press Limited, 24-28 Oval Road, London NW1 7DX, Great Britain. (1988). 